\title{
A Study on the Temporal Trends in the Etiology of Cirrhosis of Liver in Coastal Eastern Odisha
}

\author{
Debakanta Mishra ${ }^{1}$, Kaibalya R Dash² ${ }^{2}$, Chittaranjan Khatua ${ }^{3}$, Subhendu Panigrahi ${ }^{4}$, Prasanta K Parida ${ }^{5}$, Sambit K Behera ${ }^{6}$, \\ Rakesh K Barik ${ }^{7}$, Subhasis Pradhan ${ }^{8}$, Saroj K Sahu ${ }^{9}$, Bhaskar Thakur ${ }^{10}$, Shivaram P Singh ${ }^{11}$
}

\begin{abstract}
Background: The etiology of cirrhosis of liver is known to change with time due to various factors including awareness, preventive interventions, and lifestyle changes in society. However, there is scarce Indian data available about temporal trends in etiology of cirrhosis of liver. Hence, the aim of this study was to study the temporal trends in the etiology of cirrhosis of liver.

Materials and methods: This is a retrospective study conducted in the Department of Gastroenterology, Srirama Chandra Bhanja Medical College and Hospital, Cuttack, from January 2005 to December 2017. Data were collected from hospital records of all patients admitted to the Gastroenterology unit. A Poisson regression model was used to compare the hospitalization rate for different etiologies of cirrhosis of liver. All data were analyzed using Stata version 5.1 software.

Results: A total of 4,331 hospitalized patients of cirrhosis of liver were included in the analysis, of whom 2,742 (63.3\%) had alcohol-related cirrhosis, $858(19.8 \%)$ had viral hepatitis-related cirrhosis, and $731(16.9 \%)$ had cirrhosis of liver due to nonalcohol and nonviral causes. The proportion of alcohol-related cirrhosis was increased by $26 \%$ from 2005 to 2017 (RR 1.26, $p$ for trend $<0.001$ ). Though there were minimal ups and downs observed in the admission rate of viral hepatitis-related liver cirrhosis during later years, this was remarkably reduced by $73 \%$ (RR $0.27, p$ for trend $<0.001$ ) in the year 2017 at the end of the study. Similarly, the proportion of cirrhosis due to nonalcohol and nonviral causes decreased by $26 \%$ (RR 0.74, $p$ for trend <0.001) by 2017.

Conclusion: Alcohol is the most common cause of cirrhosis of liver and the burden of alcohol-related cirrhosis is significantly increasing in comparison to other causes including viral infection, nonalcoholic steatohepatitis (NASH), and autoimmune hepatitis.

Keywords: Alcohol, Cirrhosis of liver, Nonalcoholic steatohepatitis, Viral hepatitis.

Euroasian Journal of Hepato-Gastroenterology (2020): 10.5005/jp-journals-10018-1312
\end{abstract}

\section{INTRODUCTION}

Chronic liver disease (CLD) is a major cause of mortality and morbidity worldwide, accounting for approximately 2 million deaths per year. Moreover, there has been a $46 \%$ increase in cirrhosis mortality in the world from 1980 to $2013 .{ }^{1}$ Cirrhosis of liver, a final pathway for different types of CLDs is defined as diffuse fibrosis of liver parenchyma and the conversion of normal liver architecture into structurally abnormal nodules. ${ }^{2,3}$ Currently, cirrhosis and liver cancer cause 1.16 million and 788,000 deaths per year globally, respectively, making them the 11th and 16th most common causes of death, respectively. ${ }^{4}$ Together, they are responsible for $3.5 \%$ of all deaths worldwide. ${ }^{4}$ The major causes of cirrhosis of liver are alcohol and viral hepatitis. ${ }^{5,6}$ Other causes of liver cirrhosis include autoimmune diseases, ${ }^{7}$ fatty liver disease, and several inherited metabolic disorders. ${ }^{8}$ In India too, cirrhosis of liver is a major health problem. According to the latest WHO data published in 2017, liver disease deaths in India reached 259,749 or $2.95 \%$ of total deaths, accounting for one-fifth (18.3\%) of all cirrhosis deaths globally. ${ }^{1,9}$ With the rapidly growing economy and changes in lifestyle and nutrition, it is presumed that the etiological factors of liver cirrhosis in India might have changed over the past few years. It has been reported that in India, alcohol consumption increased by 55\% from 1992 to 2012 with doubling of per capita consumption between 2005 and 2016. ${ }^{10}$ Singh et al. in a study from eastern India reported that $50 \%$ of the patients with alcoholic liver disease started drinking before the legal age of drinking. ${ }^{11}$ At the same time, vaccination and effective treatment options have become available against viral hepatitis. Besides,
${ }^{1}$ Department of Gastroenterology, Institute of Medical Sciences and Sum Hospital, Bhubaneswar, Odisha, India

2,4-9,11 Department of Gastroenterology, Srirama Chandra Bhanja Medical College and Hospital, Cuttack, Odisha, India

${ }^{3}$ Department of General Medicine, MKCG Medical College, Berhampur, Odisha, India

${ }^{10}$ Division of Biostatistics, Kalinga Institute of Medical Sciences (KIMS), KIIT University, Bhubaneswar, Odisha, India

Address reprint requests to: Shivaram $P$ Singh, Department of Gastroenterology, Srirama Chandra Bhanja Medical College and Hospital, Cuttack, Odisha, India, Phone: +91 6712505466, e-mail: scb_ gastro_dept@hotmail.com

How to cite this article: Mishra D, Dash KR, Khatua C, et al. A Study on the Temporal Trends in the Etiology of Cirrhosis of Liver in Coastal Eastern Odisha. Euroasian J Hepato-Gastroenterol 2020;10(1):1-6.

Source of support: Nil

Conflict of interest: None

nonalcoholic fatty liver disease (NAFLD) which was considered to be the most common liver disease in the western countries affecting approximately $20-30 \%$ of the general population ${ }^{12,13}$ is also increasing alarmingly in India assuming pandemic proportions with a prevalence rate of $14.6-42 \%$ due to the epidemics of obesity and metabolic syndrome in the last two decades. ${ }^{14}$ In the light of the recent changes in the risk factors associated with cirrhosis of liver, there might be changes in the etiological spectrum of cirrhosis of liver over the years. Although the etiological spectrum of CLD in India has been studied previously, ${ }^{15-29}$ most of these 
studies are cross-sectional studies. There are very scarce data on the trend of etiology of cirrhosis of liver over a period of time. India being a resource-constrained region, knowledge about the trends in etiology is highly essential for effective formulation of health policies especially for resource mobilization and allocation of funds for planning preventive measures since most of the causes of cirrhosis of liver are preventable. Hence, the aim of the study was to analyze the temporal trend in the etiologies of cirrhosis of the liver.

\section{Materials and Methods}

Data were obtained from hospital records of all patients admitted to the Department of Gastroenterology, Srirama Chandra Bhanja Medical College and Hospital, Cuttack, Odisha. As this is the only gastroenterology department in state run medical colleges of Odisha, it receives patients not only from every region of Odisha but also from the neighboring states of West Bengal and Jharkhand.

\section{Study Patients}

We obtained hospitalization records for the years 2005-2017. We selected the first hospitalization record of each patient as study record. We also recorded the total number of admissions in the Gastroenterology Department in each year. As this was a retrospective study, no consent was taken but the patient's identities were concealed. The study was approved by the 'Institutional Ethics Committee'.

\section{Definitions Used in This Study \\ Cirrhosis of Liver}

Cirrhosis of liver with portal hypertension was diagnosed based on standard clinical feature (presence of ascites, shrunken liver, splenomegaly, and dilated epigastric veins), radiological evidence (shrunken liver, dilated portal vein with periportal, or other collaterals), and/or endoscopic evidence (presence of esophageal/ gastric/ectopic varices). ${ }^{30}$

Etiology of cirrhosis of liver was ascertained according to standard clinical protocols. For the purpose of this study, chronic hepatitis B was defined as a positive test for hepatitis B surface antigen. Similarly, individuals with positive HCV antibody were defined as hepatitis $C$ positive. ALD was presumed in subjects who reported excessive alcohol use (60-80 $\mathrm{g}$ alcohol per day for men, 40-60 g per day for women for 10 years or more) and had increased serum aminotransferase levels without other causes of liver disease. Subjects were presumed to have nonalcoholic steatohepatitis (NASH)-related cirrhosis, if they had cirrhosis along with diabetes mellitus or obesity $(\mathrm{BMI}>25)$ in the absence of evidence of any other etiology, such as, excessive alcohol use, increased transferrin saturation, or a positive hepatitis B or hepatitis $\mathrm{C}$ test. Iron overload was defined as transferrin saturation $>50 \%$. Other etiologies like Wilson's disease, autoimmune hepatitis, and primary biliary cirrhosis were diagnosed according to standard guidelines. In patients where no etiology could be ascertained were labeled as cryptogenic cirrhosis. For analysis of trend, patients of cirrhosis of the liver were grouped into three groups according to their etiology, the first group included patients with alcoholic liver disease, the second group included viral hepatitis, and the third group included all other causes of cirrhosis of liver like nonalcoholic fatty liver disease, autoimmune hepatitis, and cryptogenic cirrhosis. The percentage of each etiology among all patients of cirrhosis and all patients admitted to gastroenterology department was calculated.

\section{Statistical Methods}

All the categorical variables were presented as frequency $(n)$ and percentage (\%), and the continuous parameters were presented as mean \pm standard deviation (SD). A Poisson regression model was used to estimate hospitalization rate ratio (RR). For the Poisson regression model, the ratios of various etiologies [numbers of patients with alcoholic liver disease (first group), viral hepatitis (second group), and other causes of cirrhosis (third group)] as numerator and the numbers of all patients admitted to gastroenterology department as denominator were treated as dependent variables. Year was treated as an independent variable with 2005 as the reference year. All the statistical analyses were carried out by using standard statistical software Stata version 5.1 (Stata Corp LP, College Station, Texas, USA). In Stata software, a command poisson with option IRR was used to estimate hospitalization rate ratio (RR). A $p$ value of less than 0.01 was considered as statistically significant.

\section{Results}

Between the years 2005 and 2017, a total of 16,902 patients were hospitalized in the gastroenterology ward. Out of which, 4,331 patients were diagnosed to have cirrhosis of liver and were included in the analysis, of whom 2,742 (63.3\%) had alcohol-related cirrhosis, 858 (19.8\%) had viral hepatitis-related cirrhosis, and 731 (16.9\%) had cirrhosis of liver due to nonalcohol and nonviral causes (Fig. 1). The mean age of the study subjects was $46.35 \pm 13.22$ years. Majority of the cirrhosis patients were male, i.e., 3,663 out of 4,331 (84.6\%). Among the patients with alcohol-related cirrhosis, $97.5 \%$ were male, and among patients with viral hepatitis-related cirrhosis, $69.9 \%$ were male (Table 1). However, among the patients with other causes of cirrhosis, only $53 \%$ were male.

The proportion of patients with alcohol-related cirrhosis increased with time from $37 \%$ in 2005 to $74.2 \%$ in 2011 but later decreased to $62.1 \%$ in 2017 (Table 2 and Fig. 2). However, the proportion of patients with viral hepatitis-related cirrhosis had a decreasing trend with a decrease in the proportion from $39.4 \%$ in 2005 to $14.6 \%$ in 2017 . Further during the same period, the proportion of patients with other nonalcoholic and nonviral hepatitis-related cirrhosis also decreased from 23.6\% in 2005 to $9.1 \%$ in 2011 with subsequent increase to $23.3 \%$ in 2017.

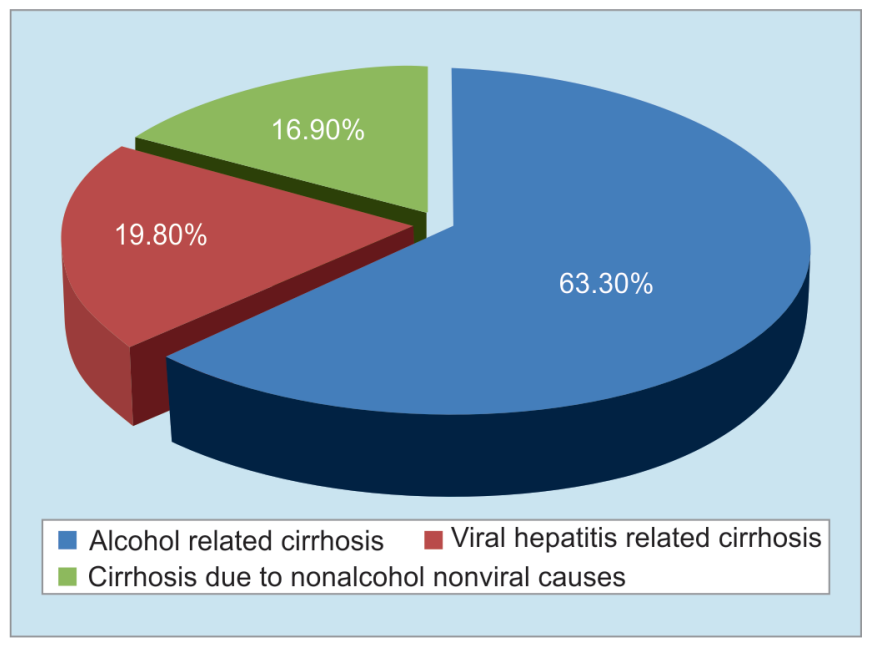

Fig. 1: Distribution of different etiologies of cirrhosis of liver 
Table 1: Sex distribution among different etiologies of cirrhosis of liver

\begin{tabular}{|c|c|c|c|c|c|c|}
\hline \multirow[b]{2}{*}{ Sex } & \multicolumn{2}{|c|}{$\begin{array}{l}\text { Alcohol-related cirrhosis } \\
(n=2,742)\end{array}$} & \multicolumn{2}{|c|}{$\begin{array}{l}\text { Viral hepatitis-related cirrhosis } \\
\qquad(n=858)\end{array}$} & \multicolumn{2}{|c|}{$\begin{array}{l}\text { Cirrhosis due to nonalcohol and nonviral } \\
\text { causes }(n=731)\end{array}$} \\
\hline & Number & $\%$ & Number & $\%$ & Number & $\%$ \\
\hline Male & 2,675 & 97.55 & 600 & 69.9 & 388 & 53.07 \\
\hline Female & 67 & 2.45 & 258 & 30.1 & 343 & 46.93 \\
\hline
\end{tabular}

Table 2: Yearwise distribution of etiologies of cirrhosis of liver

\begin{tabular}{|c|c|c|c|c|c|c|}
\hline \multirow[b]{2}{*}{ Year } & \multicolumn{2}{|c|}{ Alcohol-related cirrhosis } & \multicolumn{2}{|c|}{ Viral hepatitis-related cirrhosis } & \multicolumn{2}{|c|}{ Cirrhosis due to nonalcohol and nonviral causes } \\
\hline & Number & $\%$ & Number & $\%$ & Number & $\%$ \\
\hline 2005 & 47 & 37 & 50 & 39.40 & 30 & 23.60 \\
\hline 2006 & 61 & 48 & 43 & 33.90 & 23 & 18.10 \\
\hline 2007 & 64 & 53.80 & 23 & 19.30 & 32 & 26.90 \\
\hline 2008 & 108 & 66.70 & 29 & 17.90 & 25 & 15.40 \\
\hline 2009 & 90 & 63.80 & 32 & 22.70 & 19 & 13.50 \\
\hline 2010 & 190 & 61.50 & 70 & 22.70 & 49 & 15.90 \\
\hline 2011 & 285 & 74.20 & 64 & 16.70 & 35 & 9.10 \\
\hline 2012 & 236 & 71.50 & 48 & 14.50 & 46 & 13.90 \\
\hline 2013 & 247 & 69.20 & 60 & 16.80 & 50 & 14.00 \\
\hline 2014 & 279 & 63.70 & 92 & 21 & 67 & 15.30 \\
\hline 2015 & 319 & 63.70 & 110 & 22 & 72 & 14.40 \\
\hline 2016 & 390 & 60.00 & 137 & 21.10 & 123 & 18.90 \\
\hline 2017 & 426 & 62.10 & 100 & 14.60 & 160 & 23.30 \\
\hline
\end{tabular}

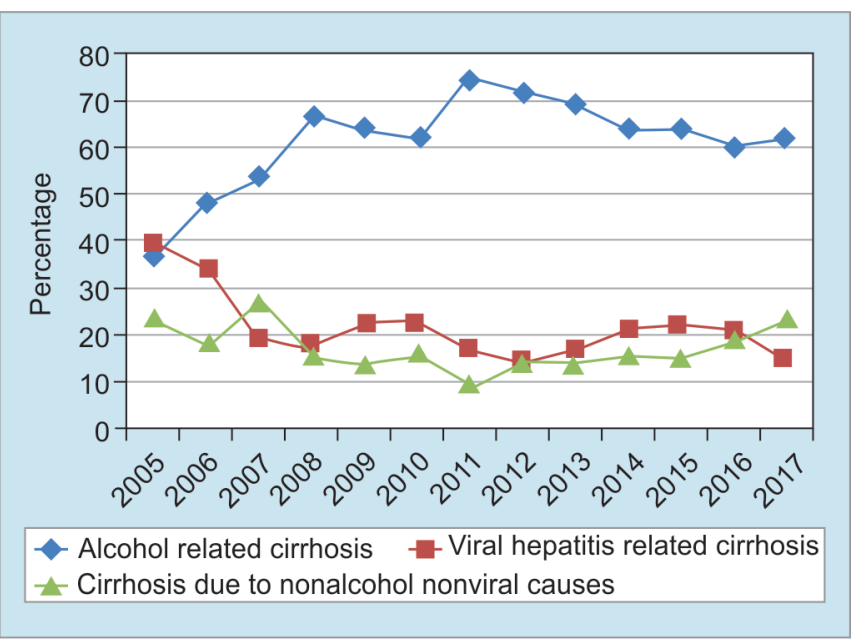

Fig. 2: Etiological trend of cirrhosis of liver

Table 3 shows the Poisson's regression analysis of percentage of each etiology among all patients admitted to the Gastroenterology unit. As we see the trend of alcoholic-related cirrhosis, considering 2005 as a reference year, admission rate had been increased up to 2008 (95\%), but decreased in subsequent year of 2009 (still 68\% increased from the reference period), but again increased during 2010 and 2011. As observed in 2012 and onward, admission rate in gastroenterology seemed to be decreasing but still remained higher than the reference year. The proportion of alcohol-related cirrhosis increased by 26\% from 2005 to 2017 (RR 1.26, p for trend $<0.001)$. Keeping 2005 as a reference year, although there were ups and down observed in the admission rate of viral hepatitis-related cirrhosis of liver during later years, it was remarkably reduced by $73 \%$ (RR 0.27, $p$ for trend $<0.001$ ) in the last year of 2017. Similarly,
Table 3: Yearwise hospitalization rate ratios due to different etiologies of cirrhosis of liver

\begin{tabular}{llll}
\hline Year & $\begin{array}{l}\text { Viral hepatitis- } \\
\text { cirrhosis, } R R\end{array}$ & $\begin{array}{l}\text { Cirrhosis due to } \\
\text { related cirrhosis, } \\
\text { RR }\end{array}$ & $\begin{array}{l}\text { nonalcohol and } \\
\text { nonviral causes, } R R\end{array}$ \\
\hline 2005 & Reference year & & \\
2006 & 1.27 & 0.84 & 0.75 \\
2007 & 1.30 & 0.44 & 1.02 \\
2008 & 1.95 & 0.49 & 0.70 \\
2009 & 1.68 & 0.56 & 0.55 \\
2010 & 1.79 & 0.62 & 0.72 \\
2011 & 1.91 & 0.40 & 0.36 \\
2012 & 1.46 & 0.28 & 0.44 \\
2013 & 1.23 & 0.28 & 0.39 \\
2014 & 1.17 & 0.36 & 0.44 \\
2015 & 1.15 & 0.37 & 0.40 \\
2016 & 1.35 & 0.44 & 0.66 \\
2017 & 1.26 & 0.27 & 0.74 \\
$p$ for trend & $<0.001$ & $<0.001$ & $<0.001$ \\
\hline
\end{tabular}

$\mathrm{RR}$, hospitalization rate ratios, estimated by Poisson regression model.

the proportion of cirrhosis of liver due to nonalcohol and nonviral causes decreased by 26\% (RR 0.74, $p$ for trend <0.001) by 2017.

Table 4 shows the distribution of hepatitis $B$ and hepatitis $C$ as the etiology of cirrhosis in the viral group. Among the patients with cirrhosis due to viral causes, hepatitis C was responsible in $5.5 \%$ of patients in 2005 and in $0.7 \%$ of patients in 2017.

\section{Discussion}

Using the hospital records from the gastroenterology unit of a tertiary care hospital in Odisha, we found that during the period 
Time Trends in Causes of Liver Cirrhosis

Table 4: Distribution of hepatitis $B$ and hepatitis $C$ in the viral group

\begin{tabular}{|c|c|c|c|c|c|c|}
\hline & \multicolumn{3}{|c|}{ Hepatitis B-related cirrhosis } & & \multicolumn{2}{|c|}{ Viral hepatitis-related cirrhosis } \\
\hline 2005 & 43 & $33.9 \%$ & & & 50 & $39.40 \%$ \\
\hline 2006 & 38 & $30 \%$ & 5 & $3.9 \%$ & 43 & $33.90 \%$ \\
\hline 2007 & 23 & $19.30 \%$ & 0 & $0.00 \%$ & 23 & $19.30 \%$ \\
\hline 2008 & 27 & $16.7 \%$ & 2 & $1.2 \%$ & 29 & $17.90 \%$ \\
\hline 2009 & 28 & $19.9 \%$ & 4 & $2.8 \%$ & 32 & $22.70 \%$ \\
\hline 2010 & 66 & $21.4 \%$ & 4 & $1.3 \%$ & 70 & $22.70 \%$ \\
\hline 2011 & 58 & $15.1 \%$ & 6 & $1.6 \%$ & 64 & $16.70 \%$ \\
\hline 2012 & 44 & $13.3 \%$ & 4 & $1.2 \%$ & 48 & $14.50 \%$ \\
\hline 2013 & 54 & $15.1 \%$ & 6 & $1.7 \%$ & 60 & $16.80 \%$ \\
\hline 2014 & 89 & $20.3 \%$ & 3 & $0.7 \%$ & 92 & $21 \%$ \\
\hline 2015 & 104 & $20.8 \%$ & 6 & $1.2 \%$ & 110 & $22 \%$ \\
\hline 2016 & 131 & $20.2 \%$ & 6 & $0.9 \%$ & 137 & $21.10 \%$ \\
\hline 2017 & 95 & $13.9 \%$ & 5 & $0.7 \%$ & 100 & $14.60 \%$ \\
\hline
\end{tabular}

from 2005 to 2017, 4,331 patients had been hospitalized for cirrhosis of liver. Alcohol was the etiology for around two-thirds of the patients whereas viral hepatitis was the etiology for onefifth of the patient population. Over the years, the proportion of alcohol-related cirrhosis of liver increased and proportion of viral hepatitis-related cirrhosis declined. The proportion of patients having nonviral and nonalcohol-related cirrhosis, which included cryptogenic cirrhosis, autoimmune hepatitis, NASH-related cirrhosis, and other rare etiologies declined in the period from 2005 to 2011 but subsequently increased to $23.3 \%$. Male-to-female sex ratio was significantly higher for all etiologies of cirrhosis. Our study provides a trend in the etiology of cirrhosis of liver over a period of 13 years.

Our study showed that alcohol was the major cause of cirrhosis of liver in this region. Bhattacharyya et al. studied 1,000 consecutive cirrhosis patients from northeastern India and diagnosed alcoholrelated cirrhosis in $72.2 \%$ of patients. ${ }^{15}$ Sharma et al. too in a study from northern India reported alcohol to be the most common $(62.9 \%)$ etiological factor for cirrhosis. ${ }^{18}$ Pati et al. in a previous study from the same center also reported alcohol to be the cause of cirrhosis of liver in $65.04 \%$ of cases of acute on chronic liver failure. ${ }^{19}$ Multiple studies from different centers of India have reported alcohol to be the commonest cause of cirrhosis with prevalence ranging from $30 \%$ to $70 \%$ of all cases of cirrhosis. ${ }^{20-26}$ Patil et al. reported that the main etiology of cirrhosis was ethanolrelated (38\%) followed by NASH-related (24\%), hepatotropic viruses (22\%), and cryptogenic cirrhosis (17\%). ${ }^{27}$ However, contrary to most reports, Ray from Kolkata reported that alcohol was overall the third most common cause of CLD after cryptogenic (36.5\%) and viral hepatitis (33.2\%) for the entire period of study from 2003 to 2011; however, this study too demonstrated that over time, during the last year of their study period, alcohol emerged as the commonest cause of cirrhosis of the liver. ${ }^{28}$ In another recent multicenter study, Mukherjee et al. who studied the etiology and mode of presentation of CLD in different centers of India reported that though alcohol was commonest cause of cirrhosis of liver (34.3\%), chronic hepatitis $B$ was the commonest cause of noncirrhotic CLD. ${ }^{29}$

Another important finding from our study is the increasing rate of alcohol-related cirrhosis over time, although the rate seems to have stabilized at around $60 \%$ during the last three years of our study period. Besides, from the regression analysis, we found that the proportion of patients with alcohol-related cirrhosis among all patients admitted to the gastroenterology unit had increased by $26 \%$ from 2005 to 2017. Ray from Kolkata had also noted a similar rise in the rate of alcohol-related CLD in his study - from 22\% in 2003 to $42 \%$ in $2011 .{ }^{28}$ Further, Thomson et al. from England also reported that the hospital admissions and mortality from CLD were increasing and alcohol-induced liver disease was making a major contribution to it. ${ }^{31}$ Bao et al. too reported a rising trend of alcoholrelated cirrhosis in China. ${ }^{32}$ Similarly, Kim et al. too observed in a population-based study from USA that mortality from alcoholic liver disease increased from 2007 to $2016 . .^{33}$ This rising trend of alcohol-related cirrhosis of liver has been explained by earlier age of acquiring alcoholism, an increasing per capita intake of alcohol, and increasing trends of "at risk" drinking. 10,34

Viral hepatitis was the second most common etiological factor in our study, which is similar to the findings of Sharma et al. ${ }^{18}$ However, some studies from India have found it to be the most common cause of cirrhosis of liver, but these studies are 2-3 decades old and there have been rapid changes in the epidemiology in the last few decades. ${ }^{16,17}$ Recently, a study from the same group had reported alcohol to be the commonest cause of cirrhosis of the liver. ${ }^{27}$ Viral hepatitis as an etiological factor for cirrhosis has clearly shown a decreasing trend in our study. This observation of decreasing trend is similar to the observations of Ray, ${ }^{28}$ Bao et al., ${ }^{32}$ and Kim et al. ${ }^{33}$ Chiang et al. studied the 30 -year outcomes of the National Hepatitis B Immunization Program in Taiwan and reported a continuous decline in age- and sex-adjusted rate ratios of CLD and hepatocellular carcinoma (HCC) mortality and HCC incidence for birth cohorts born after implementation of the program. ${ }^{35}$ However in India, universal vaccination against hepatitis B was included in the National Immunization Schedule in 2007-2008. ${ }^{36}$ Hence, this decrease in viral hepatitis as an etiology of cirrhosis of liver obviously cannot be explained due to the availability of vaccination against hepatitis B or blood bank screening, since it would take decades for these interventions to impact cirrhosis prevalence. Moreover, a previous study from the same center by Misra et al. had reported a vaccination rate of only $20 \%$ among 682 individuals and $30 \%$ among the children of the study subjects. ${ }^{37}$ This decreasing trend of viral infection as etiological factor of cirrhosis may however be explained due to the rising trend of alcohol as the etiology of cirrhosis of liver. When the viral group was divided into cirrhosis due to chronic 
hepatitis $B$ and cirrhosis due to chronic hepatitis $C$, we found that the prevalence of hepatitis $B$ as the etiology is much higher than hepatitis $C$. Chronic hepatitis $C$ was the etiology of $5.5 \%$ of patients in 2005 which decreased to $0.7 \%$ in 2017. Mukherjee et al, in their multicenter study reported hepatitis $C$ to be the causative factor in $21.6 \%$ of CLD patients from all over India whereas it was the etiological factor in $9.4 \%$ of CLD patients from eastern India. ${ }^{29}$ The prevalence of hepatitis $C$ as an etiological factor of cirrhosis in our region was even lower than the prevalence reported by Mukherjee et al. and it showed a decreasing trend.

In our study, we defined nonalcohol and nonviral hepatitisrelated cirrhosis to include every other etiology except viral hepatitis and alcohol. However, our definition is ambiguous and, therefore, the trend of the disease may be affected by various causes. There was a decreasing trend of this group from 2005 to 2011, with an increasing trend in subsequent years. Since the proportion of causes of cirrhosis is only a relative statistics, it can be affected by a variation in the proportion of other causes of cirrhosis. Besides, since this group also includes cirrhosis due to nonalcoholic fatty liver disease, the temporal trend might be a reflection of the rising trend of NAFLD in the population. ${ }^{38}$

Our study is fraught with significant limitations. Being a single center, retrospective study is a major limitation of our study; however, since our center is the only tertiary care hospital with gastroenterology unit in the state catering to patients from all socioeconomic status, the results of our study can be extrapolated to the whole region. Without histology, some patients might have wrongly been labeled as cryptogenic cirrhosis. Further, we have not studied the mortality rates due to the etiological factors which could have given more information.

\section{Conclusion}

Alcohol is the major cause of cirrhosis of liver in coastal eastern India, and there is a rising trend in the burden of alcohol-related cirrhosis of liver. The incidence of nonalcohol and nonviral hepatitis-related cirrhosis also appears to be rising and this group includes patients with NAFLD-related cirrhosis. Since all of these major etiological factors are preventable causes of cirrhosis of liver, the government and the professional medical and Hepatology societies should come together to formulate effective strategies for prevention of cirrhosis of liver.

\section{References}

1. Mokdad AA, Lopez AD, Shahraz S, et al. Liver cirrhosis mortality in 187 countries between 1980 and 2010: a systematic analysis. BMC Med 2014;12(1):145. DOI: 10.1186/s12916-014-0145-y.

2. Anthony PP, Ishak KG, Nayak NC, et al. The morphology of cirrhosis: Definition, nomenclature, and classification. Bull World Health Organ 1977;55(4):521-540.

3. Hytiroglou P, Snover DC, Alves V, et al. Beyond "cirrhosis": a proposal from the International Liver Pathology Study Group. Am J Clin Pathol 2012;137(1):5-9. DOI: 10.1309/AJCP2T2OHTAPBTMP.

4. WHO. Global health estimates 2015: deaths by cause, age, sex, by country and by region, 2000-2015. Geneva: World Health Organization; 2016.

5. Dufour MC. Alcoholic liver disease. Gl epidemiology Talley NJ, Locke GR, Saito YA 1st edn., Massachusetts: Blackwell Publishing Inc; 2007. pp. 231-237.

6. Perz JF, Armstrong GL, Farrington LA, et al. The contributions of hepatitis $B$ virus and hepatitis $C$ virus infections to cirrhosis and primary liver cancer worldwide. J Hepatol 2006;45(4):529-538. DOI: 10.1016/j.jhep.2006.05.013.

7. Selmi C, Bowlus $\mathrm{CL}$, Gershwin ME, et al. Primary biliary cirrhosis. Lancet 2011;377(9777):1600-1609. DOI: 10.1016/S0140-6736(10)61965-4.

8. Brunt EM. Non-alcohol fatty liver disease. MacSween's Pathology of the Liver Burt 5th edn., Philadelphia, PA: Churchill Livingstone; 2007. pp. 367-397.

9. World Life Expectancy. India: Liver Disease. In: World Health Rankings. World Life Expectancy. Available at: https://www. worldlifeexpectancy.com/india-liver-disease, Accessed on 9 November 2018.

10. OECD (2017), "Alcohol consumption among adults", in Health at a Glance 2017: OECD Indicators, OECD Publishing, Paris 10.1787/ health_glance-2017-17-en.

11. Singh SP, Padhi PK, Narayan J, et al. Socioeconomic impact of alcohol in patients with alcoholic liver disease in eastern India. Indian J Gastroenterol 2016;35(6):419-424. DOI: 10.1007/s12664-016-0699-z.

12. Younossi ZM, Koenig $A B$, Abdelatif $D$, et al. Global epidemiology of nonalcoholic fatty liver disease - meta-analytic assessment of prevalence, incidence, and outcomes. Hepatology. 2016;64(1):73-84. DOI: 10.1002/hep.28431.

13. Angulo P. Gl epidemiology: nonalcoholic fatty liver disease. Aliment Pharmacol Ther 2007;25(8):883-889. DOI: 10.1111/j.13652036.2007.03246.x.

14. Pati GK, Singh SP. Nonalcoholic fatty liver disease in South Asia. Euroasian J Hepato-Gastroenterol 2016;6(2):154-162. DOI: 10.5005/ jp-journals-10018-1189.

15. Bhattacharyya M, Barman NN, Goswami B. Survey of alcoholrelated cirrhosis at a tertiary care center in North East India. Indian J Gastroenterol 2016;35(3):167-172. DOI: 10.1007/s12664-016-0651-2.

16. Sarin SK, Chari S, Sundaram KR, et al. Young v adult cirrhotics: a prospective, comparative analysis of the clinical profile, natural course and survival. Gut 1988;29(1):101-107. DOI: 10.1136/gut.29.1.101.

17. Ray G, Ghoshal UC, Banerjee PK, et al. Aetiological spectrum of chronic liver disease in eastern India. Trop Gastroenterol 2000;21(2):60-62.

18. Sharma B, Marwah R, Raina S, et al. A study on the etiology of cirrhosis of liver in adults living in the hills of Himachal Pradesh, India. Trop Gastro 2017;37(1):37-41. DOI: 10.7869/tg.317.

19. Pati GK, Singh A, Misra B, et al. Acute-on-chronic liver failure (ACLF) in Coastal Eastern India: "A Single-Center Experience". J Clin Exp Hepatol 2016;6(1):26-32. DOI: 10.1016/j.jceh.2015.08.002.

20. Ramanathan S, Khandelwal N, Kalra N, et al. Correlation of HVPG level with CTP score, MELD Score, ascites, size of varices, and etiology in cirrhotic patients. Saudi J Gastroenterol 2016;22(2):109-115. DOI: 10.4103/1319-3767.164185.

21. Kumar R, Kumar P, Saxena KN, et al. Vitamin D status in patients with cirrhosis of the liver and their relatives - A case control study from North India. Indian J Gastroenterol 2017;36(1):50-55. DOI: 10.1007/ s12664-017-0727-7.

22. Bishnu S, Ahammed SM, Sarkar A, et al. Effects of atorvastatin on portal hemodynamics and clinical outcomes in patients with cirrhosis with portal hypertension: a proof-of-concept study. Eur J Gastroenterol Hepatol 2018;30(1):54-59. DOI: 10.1097/MEG.0000000000001006.

23. Shah AS, Amarapurkar DN. Natural history of cirrhosis of liver after first decompensation: A prospective study in India. J Clin Exp Hepatol 2018;8(1):50-57. DOI: 10.1016/j.jceh.2017.06.001.

24. Punekar P, Sharma AK, Jain A. A study of thyroid dysfunction in cirrhosis of liver and correlation with severity of liver disease. Indian J Endocrinol Metab 2018;22(5):645-650.DOI: 10.4103/ijem.IJEM_25_18.

25. Kirnake V, Arora A, Sharma P, et al. Non-invasive aspartate aminotransferase to platelet ratio index correlates well with invasive hepatic venous pressure gradient in cirrhosis. Indian J Gastroenterol 2018;37(4):335-341. DOI: 10.1007/s12664-018-0879-0.

26. Kulkarni S, Sharma M, Rao PN, et al. Acute on chronic liver failurein-hospital predictors of mortality in ICU. J Clin Exp Hepatol 2018;8(2):144-155. DOI: 10.1016/j.jceh.2017.11.008. 
27. Patil AG, Bihari C, Shewade HD, et al. Decreased protein $C$ function predicts mortality in patients with cirrhosis. Int J Lab Hematol 2018;40(4):466-472. DOI: 10.1111/ijlh.12836.

28. Ray G. Trends of chronic liver disease in a tertiary care referral hospital in eastern India. Indian J Public Health 2014;58(3):186-194. DOI: 10.4103/0019-557X.138630.

29. Mukherjee PS, Vishnubhatla S, Amarapurkar DN, et al. Etiology and mode of presentation of chronic liver diseases in India: a multi centric study. PloS one. 2017(10):12. DOI: 10.1371/journal.pone.0187033.

30. Schuppan D, Afdhal NH. Liver cirrhosis. Lancet 2008;371(9615): 838-851. DOI: 10.1016/S0140-6736(08)60383-9.

31. Thomson SJ, Westlake S, Rahman TM, et al. Chronic liver disease--an increasing problem: a study of hospital admission and mortality rates in England, 1979-2005, with particular reference to alcoholic liver disease. Alcohol Alcohol. 2008;43(4):416-422. DOI: 10.1093/alcalc/ agn020.

32. Bao $X-Y, X u B-B$, Fang $K$, et al. Changing trends of hospitalisation of liver cirrhosis in Beijing, China. BMJ Open Gastro 2015;2(1):e000051. DOI: 10.1136/bmjgast-2015-000051.
33. Kim D, Li AA, Gadiparthi C, et al. Changing trends in etiology-based annual mortality from chronic liver disease, from 2007 through 2016. Gastroenterology 2018;155(4):1154-1163. DOI: $10.1053 / \mathrm{j}$ .gastro.2018.07.008.

34. Prasad R. Alcohol use on the rise in India. Lancet 2009;373(9657):17-18. DOI: 10.1016/S0140-6736(08)61939-X.

35. Chiang CJ, Yang YW, You SL, et al. Thirty-year outcomes of the national hepatitis B immunization program in Taiwan. JAMA 2013;310(9): 974-976. DOI: 10.1001/jama.2013.276701.

36. Govt of India. Operational guidelines for the introduction of Hepatitis B vaccine in UIP of India. New Delhi: Mo HFW, Govt of India; 2008.

37. Singh S, Misra B, Panda C, et al. Study on awareness about Hepatitis B viral infection in coastal Eastern India. Hep B Annual 2009;6(1):19-28. DOI: 10.4103/0972-9747.76902.

38. Wong MCS, Huang JLW, George J, et al. The changing epidemiology of liver diseases in the Asia-Pacific region. Nat Rev Gastroenterol Hepatol 2019;16(1):57-73. DOI: 10.1038/s41575-0180055-0. 